$$
\text { CONF-970/52-1 }
$$

\title{
Laboratory Evaluation of \\ Dynamic Traffic Assignment Systems: \\ Requirements, Framework, and System Design
}

to be submitted for review

for presentation in

Transportation Research Board's 76th Annual Meeting

January 1997, Washington, D.C.

and

for publication in

Transportation Research Record

[*The first 3 authors listed below are in alphabetical order.]

Shaw-Pin Miaou*

Oak Ridge National Laboratory

P.O.Box 2008, Building 5500A, Oak Ridge, TN 37831-6366, USA

Phone: (423) 574-6933, Fax: (423) 574-3851, Email: PNI@ORNL.GOV

Rekha S. Pillai*

Oak Ridge National Laboratory

P.O.Box 2008, Building 4500N, Oak Ridge, TN 37831-6206, USA

Phone: (423) 574-1402, Fax: (423) 574-4747, Email: PUV@ORNL.GOV

Mike S. Summers*

Oak Ridge National Laboratory

P.O.Box 2008, Building 4500N, Oak Ridge, TN 37831-6207, USA

Phone: (423) 576-4488, Fax: (423) 574-4747, Email: X7U@ORNL.GOV

Ajay K. Rathi

Oak Ridge National Laboratory

P.O.Box 2008, Building 4500N, Oak Ridge, TN 37831-6207, USA

Phone: (423) 574-5003, Fax: (423) 574-4747, Email: AKR@ORNL.GOV

Henry C. Lieu

Federal Highway Administration

Intelligent Systems Division (HSR-11, Room T-306), Office of Safety and Traffic Operations R\&D

6300 Georgetown Pike, McLean, VA 22101-2296, USA

Phone: (703) 285-2410, Fax: (423) 285-2264, Email: HLIEU@ INTERGATE.DOT.GOV

Prepared by the

Oak Ridge National Laboratory

Oak Ridge, Tennessee 37831-6366

managed by

LOCKHEED MARTIN ENERGY RESEARCH CORP.

for the U.S. DEPARTMENT OF ENERGY

under contract DE-AC05-96OR22464 


\section{DISCLAIMER}

This report was prepared as an account of work sponsored by an agency of the United States Government. Neither the United States Government nor any agency thereof, nor any of their employees, makes any warranty, express or implied, or assumes any legal liability or responsibility for the accuracy, completeness, or usefulness of any information, apparatus, product, or process disclosed, or represents that its use would not infringe privately owned rights. Reference herein to any specific commercial product, process, or service by trade name, trademark, manufacturer, or otherwise does not necessarily constitute or imply its endorsement, recommendation, or favoring by the United States Government or any agency thereof. The views and opinions of authors expressed herein do not necessarily state or reflect those of the United States Government or any agency thereof. 


\section{DISCLAmMER}

Portions of this document may be illegible in electronic image products. Images are produced from the best available original document. 


\title{
Laboratory Evaluation of \\ Dynamic Traffic Assignment Systems: \\ Requirements, Framework, and System Design
}

\begin{abstract}
The success of Advanced Traveler Information Systems (ATIS) and Advanced Traffic Management Systems (ATMS) depends on the availability and dissemination of timely and accurate estimates of current and emerging traffic network conditions. Real-time Dynamic Traffic Assignment (DTA) systems are being developed to provide the required timely information. The DTA systems will provide faithful and coherent real-time, pre-trip, and en-route guidance/information which includes routing, mode, and departure time suggestions for use by travelers, ATIS, and ATMS. To ensure the credibility and deployment potential of such DTA systems, an evaluation system supporting all phases of DTA system development has been designed and presented in this paper. This evaluation system is called the DTA System Laboratory (DSL). A major component of the DSL is a ground-truth simulator, the DTA Evaluation System (DES).

The DES is envisioned to be a virtual representation of a transportation system in which ATMS and ATIS technologies are deployed. It simulates the driving and decision-making behavior of travelers in response to ATIS and ATMS guidance, information, and control. This paper presents (i) the major evaluation requirements for a DTA Systems, (ii) a modular modeling framework for the DES, and (iii) a distributed DES design.

The modeling framework for the DES is modular, meets the requirements, can be assembled using both legacy and independently developed modules, and can be implemented as a either a single process or a distributed system. The distributed design is extendible, provides for the optimization of distributed performance, and object-oriented design within each distributed component. A status report on the development of the DES and other research applications is also provided.
\end{abstract}

Key Words: DTA System, Laboratory testing and evaluation, Traffic Simulation, Distributed software system 


\section{Laboratory Evaluation of Dynamic Traffic Assignment Systems: Requirements, Framework, and System Design}

\section{BACKGROUND}

Intelligent Transportation Systems (ITS) are currently being developed and deployed to exploit computer and electronic communication technologies to improve the efficiency, productivity, and safety of existing transportation facilities and the environment. The success of important ITS sub-systems, such as Advanced Traveler Information Systems (ATIS) and Advanced Traffic Management Systems (ATMS), depend on the availability and dissemination of timely and accurate estimates of prevailing and emerging traffic conditions. The Federal Highway Administration (FHWA) is meeting the need for such information through their sponsorship of the development of real-time Dynamic Traffic Assignment (DTA) systems.

Based on real-time traffic and weather data from multiple surveillance systems, historical databases, together with available ATIS and ATMS guidance and control strategies, DTA systems will utilize data fusion technologies and state-of-the-art traffic estimation and prediction models to produce required information and guidance that is consistent with the prevailing and emerging traffic conditions and meet various guidance and control objectives. Furthermore, the systems will provide faithful and coherent real-time, pre-trip, and en-route guidance and information which will include route, mode, and departure time suggestions, congestion levels, and incidence warnings to travelers through ATIS and ATMS sub-systems. The DTA system, thus, forms a bridge between real-time traffic surveillance systems and ATIS and ATMS and can be considered as a principal cognitive element of ITS (Figure 1).

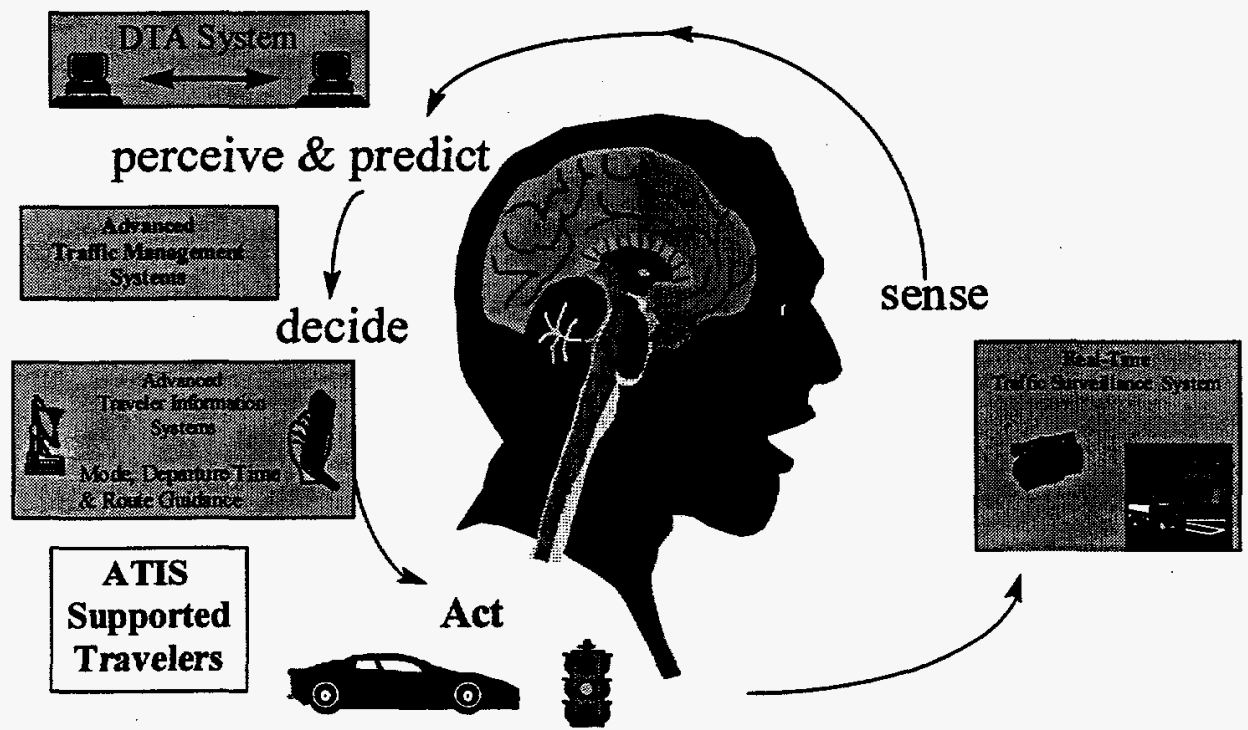

Figure 1 DTA System: The Cognitive Element of Intelligent Transportation System

While the need for DTA systems is clear, research into the best combination of models, algorithms, software architectures, and processing paradigms that will enable DTA systems to perform as required in the field is not complete. Nonetheless, it is clear from current research that a widely deployable DTA system will be a complex distributed software system. 
Oak Ridge National Laboratory (ORNL) has been designated by FHWA as the manager for the development of a deployable, real-time DTA system. ORNL's roles include

- managing contracts for the development of DTA systems and components

- evaluating alternative DTA approaches to minimize development and deployment risk

- integrating DTA system components into an effective system

- testing and evaluating the developed DTA system

The DTA system will be developed in multiple phases over several years. Phase I development will produce a functional DTA system with established potential for deployment and real-time operations. Later phases will focus on real-time operations and field-testing issues and result in a deployable, realtime DTA system.

In October 1995, ORNL concluded a competitive procurement with the award of two contracts to support the Phase I effort. The winning proposals were written by the Massachusetts Institute of Technology (MIT) and The University of Texas at Austin (UTX). Phase I work is scheduled for completion in two years.

The DTA systems being developed by MIT and UTX are based on a rolling-horizon implementation of assignment-simulation framework, uses distributed computing to support real-time operations, and support synchronization with real-time data, See reports $(1,2)$. Some of the differences between the two systems are in terms of: representations of transportation network; traffic flow models; assumptions regarding driver behavior in response to ATIS and ATMS information and guidance; approaches to real-time data synchronization; and the system design, integration, operation, guidance and control objectives.

As designated integrator and evaluator, ORNL will be responsible for performing comprehensive, costeffective evaluations of DTA systems to ensure their credibility and deployment potential. These evaluations will include functional, design, model, software, and system evaluations. ORNL is currently developing DTA testing and evaluation procedures and a design for a comprehensive evaluation system supporting all phases of DTA system development.

The comprehensive evaluation system will be referred to as the DTA System Laboratory (DSL). A major component of the DSL is a ground-truth simulator which, for the purpose of this paper, will be called the DTA Evaluation System (DES). The DES is envisioned to be a virtual representation of a transportation system in which ATMS and ATIS technologies are deployed. It is a traffic simulator that simulates the driving and decision-making behavior of travelers in response to ATIS and ATMS guidance, information, and controls and is sensitive to the time-varying physical and operational states of the traffic network and environment (e.g., lighting and weather conditions-daytime versus nighttime, snow, rain, fog, etc.).

The objective of this paper is to summarize the DTA System evaluation requirements and describe the resulting DES modeling framework and system design. This paper also provide a brief update on the status of the development of the DES. Subsequent papers and reports will address other facets of the DES and DSL, including the DTA system evaluation methodology, the DTA system evaluation data requirements, and potential for DES application in other ITS models and system development and deployment activities. 
This paper is organized as follows. Section 2 gives an overview of the DSL to provide a context for the DES. Section 3 summarizes some of the highlights of DES requirements. Section 4 describes a generic, modular, modeling framework for the development of alternate DESs. Section 5 describes the design of a distributed DES for use within the DSL. Section 6 provides a summary of the paper, a status report regarding the development of the DES, and a brief description of future research opportunities.

\section{DTA SYSTEM LABORATORY OVERVIEW}

The need for a DSL to develop and evaluate DTA systems is driven by several major considerations.

- Different regions will have different characteristics, budget constraints, control objectives, and requirements and will deploy different levels of ITS services in different ways. Because of this, DTA systems will require deployment-specific customization.

- Since ITS deployments are driven by market and infrastructure developments, they will evolve over time. Thus DTA systems will require a life time evaluation.

- DTA systems must support tight coupling with ITS sub-systems such as ATMS, ATIS, and surveillance systems. These sub-systems are still undergoing research and development. Because of this, DTA systems may have to be evaluated and re-evaluated in light of later developments.

- There are many candidate DTA systems that can be assembled and integrated using various combinations of modeling components, algorithms, and operational assumptions. Thus, there are a large number of candidate systems to be evaluated.

For example, the DTA systems being developed by MIT and UTX are different in network representation, models, behavioral and operational assumptions, and many other areas, discussed above. Given the different DTA components that might be obtained from these two systems, and from systems developed by other researchers and developers, the development of a large number of hybrid DTA systems is possible.

- DTA systems are complex, distributed, real-time information-processing systems involving human decision-making and learning processes. Therefore, DTA systems cannot be designed from first principles, but must be developed interactively and iteratively through the experimental evaluations of candidate systems in the context of multiple simulated ITS deployments.

In order to economically support the extensive DTA development, integration, and evaluation process, a DSL is required. This laboratory is envisioned to be a special purpose software environment which combines

- DTA and ITS specific software development environment that serves as a framework for software reuse, a software integration medium, and a tool-kit for the development and integration of a variety of DTA related software sub-systems

- DES that is capable of representing various transportation system configurations and infrastructures, ITS deployments, and traveler driving and decision making behavior in response to guidance/information and control.

The purpose of the ITS specific software development environment is to minimize the cost of developing and integrating multiple candidate DTA systems and required variations of the simulation test-bed. 
Figure 2 presents the DTA evaluation framework. This framework is comprised of a ground-truth simulator and simulation support sub-system components including scenario generation tools and data analysis and visualization tools.

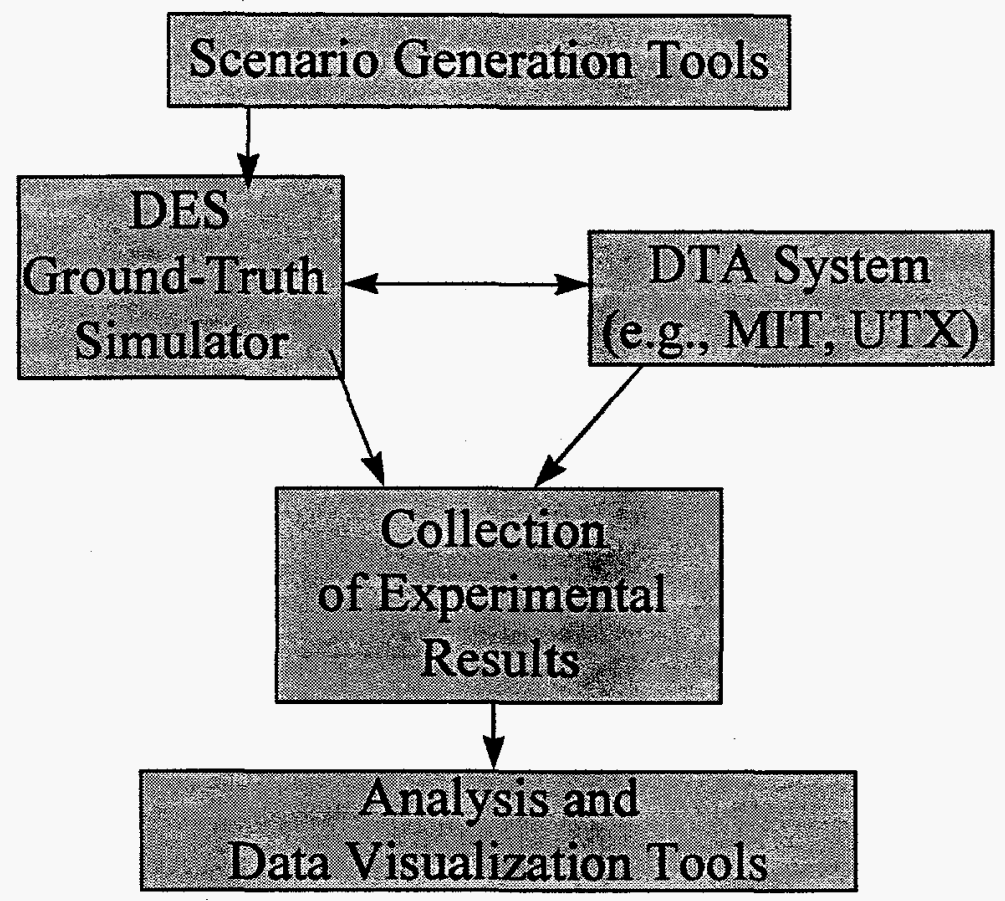

Figure 2 DTA Evaluation System (DES) Framework

\section{GROUND-TRUTH SIMULATOR REQUIREMENTS}

The ground-truth simulator is a virtual representation of a transportation system in which ATMS and ATIS technologies are deployed. The top-level functional requirements of the simulator can then be summarized as follows:

- It is required to represent the important physical and operational characteristics of the transportation network

- It should model the physical and operational characteristics of vehicle movements including ATIS equipment, vehicle dimension, occupancy, acceleration time, deceleration time, etc.

- It should model driving behavior characteristics such as car-following, lane changing, overtaking etc.

- It should model travelers' pre-trip and en-route compliance and decision-making behavior including mode, departure time, and route choices in response to different types of descriptive and prescriptive ATIS and ATMS guidance/information and controls

- It should be capable of generating and injecting various types of short-term stochastic and longterm scheduled incidents and their dynamic effects to the traffic and transportation facility capacity 
- It should capture a wide range of plausible ATMS and ATIS deployments, operational characteristics, and impacts, e.g., centralized versus decentralized Traffic Management Center's (TMC), different levels of services that could potentially be offered by ATIS providers, various types and densities of surveillance system deployments (including probe vehicles), different types of incident detection, reporting, and management systems

A detailed discussion of the functional requirements is given in (3)and (4) The highlights of a selected number of the functional requirements and related performance requirements are discussed and qualified as follows:

\section{Transportation Network Representation}

In theory, the simulator can use very detailed network representation to capture every physical and operational characteristics of the network over space and time. For example, the network representation can include the physical and operational characteristics of every street (including mainline and roadside), parking lot, driveway, and access control. In practice, the required representation is typically driven by the highest level of details under which the DTA system is required to be evaluated, as well as other implementation constraints such as time, cost, computational, and data constraints.

When the network is represented in abstract by nodes and links in simulation, the attributes of the network which are judged to have significant effects on the validity of the DTA system evaluation will have to be faithfully represented. These include those important physical and operational characteristics which have influences on driving behavior, vehicle operational performance, and traffic flows, e.g., lane additions, lane drops, and high-occupancy-vehicle (HOV) lanes, and signal controls.

Freeways, surface streets, and ramps have distinct differences in physical and operational characteristics, e.g., geometrics, design speed, and access controls. The network representation is required to capture the important features of each of these sub-networks.

\section{Modeling Dynamic Trip Demand and Distribution}

Models are required to generate the trip demand by origin and destination, mode, departure time, and even path. In the strict sense, the demand may be elastic with respect to network conditions, the information of which is disseminated through the ATIS and ATMS medium. Also, the elasticity may vary over time, both within-day and between-days.

However, at present, data in this area are rather limited. It is not possible to identify the best set of models with confidence. Instead, a suite of plausible trip making behavior models, which are flexible and extendible, should be included in the simulator. This would allow more complicated trip making decisions, such as trip-chaining, re-moding and re-destination, to be captured in future when more data becomes available. 


\section{Modeling of Route Choice Behavior in Response to ATIS and ATMS Information}

The same ATIS or ATMS information may impact drivers in a very different way. It depends not only on drivers' characteristics (such as age, gender, and driving experience), familiarity with the network and surrounding area, type of network, trip purpose, time of trip, and trip utility, but also on the credibility that the ATIS providers and ATMS have established. As a result, whether the route guidance is from the ATIS providers or from Variable Message Signs (VMS), full compliance of all drivers, with access to the information and guidance, is not guaranteed. The ability of DTA system to produce good estimation and prediction of traffic conditions is partially dependent on how good the system can model driver's response information and guidance and controls. To date, no clear consensus has been reached regarding the major elements of driver behavior modeling. As in the trip making decision modeling stated above, a suite of plausible route choice behavior models, which are flexible and extendible, should be included in the simulator. This would allow various choice behavior assumptions and types that have been proposed in the literature, such as System Optimal (SO), User Optimal/Equilibrium (UO/UE), Boundedly Rational (BR), and other utility maximizing driver behavior to be simulated and tested.

\section{Vehicle Modeling}

In addition to the physical and operational characteristics of vehicles, the simulator needs to be able to consider whether a vehicle is equipped with the ATIS equipment and, if it does, the type of ATIS equipment. The type of ATIS equipment in a vehicle determines the vehicle's ability to access, receive, and send various types of guidance and information. For example, a vehicle equipped with two way communication system, such as a probe vehicle, can both receive and transmit information on network states.

A wide range of market penetration rates for different types of ATIS equipment need to be simulated to capture the evolutionary impacts of ITS and location-specific effects that were alluded to earlier. Subtle issues such as the correlation among driver's characteristics, vehicle ownership, and ATIS equipment ownership needs to be modeled as well.

\section{Path-Based Vehicle Movement}

Modeling of vehicle movements on path is an important modeling requirement of DES. One of the functions of DTA system is to provide route guidance information that is consistent or meet various traffic control objectives. In order to evaluate different pre-trip and en-route route guidance generation strategies and algorithms, it is essential that DES be able to simulate the path movements of vehicles. The paths on which the vehicles move, could be either static, i.e., remain the same for the duration of the trip or dynamic, i.e., change as the traffic conditions change and/or new information is received by the drivers. A path chosen at the beginning of a trip or changed by a driver en-route is determined by the route choice behavior models discussed earlier.

\section{Incident Generation, Detection, Reporting, and Management Modeling}

Incidents impact supply and may also impact demand, depending on traffic condition. The ability to accurately estimate and predict network states and generate guidance information to alleviate congestion is highly dependent on the ability to detect, report, and manage the incidents. The detection, reporting, and management of incidents can, however, be achieved through very different means (e.g., traffic detectors, video camera, phones, highway patrol), and can involve complicated institutional and technological issues. 
To evaluate the DTA system's ability to effectively utilize incident information as it becomes available in real-time, the DES must be capable of generating incidents, both planned and randomly occurring, of different severities and durations and at different locations and times. Thus, the simulator should be capable of dynamically generating random, short-term incidents based on link geometry, current simulated traffic conditions (including nearby incidents), and weather conditions.

\section{Surveillance Systems Modeling}

Surveillance systems are the eyes and ears of a DTA system. The traffic can be expected to be highly dynamic and stochastic under ATMS and ATIS. For a real-time DTA system to perform well, its ability to synchronize the system with various sources of real-time surveillance system data, including incident data, is key.

To be able to evaluate how effectively surveillance system information is utilized by a DTA system, the DES needs to have the capability to model different types of surveillance systems and the type of information generated by them at different levels of deployment. The surveillance system modeling should take into consideration the operational parameters, including location of the system, area of coverage, type of coverage, and operational limitations. Some of the surveillance systems that the DES must be able to model are vehicle loop detectors, radar, over-height detectors, probe vehicles, widearea surveillance systems, cellular phones, and other communication technologies.

\section{ATMS Modeling}

One of the most important characteristics of the transportation system that a DTA system must capture is the dynamic supply-demand interactions. The ATMS components (signal control systems, incident management systems, VMS, etc.) attempt to control the supply side of the transportation system (intersection capacity, link capacity, etc.) in response to varying demand. A DTA system should capture the changes in supply over time in order to estimate the changes and impact on demand and the transportation system states. In order to evaluate a DTA system, the DES should be able to model and simulate an ATMS to analyze the modeling of supply-demand interactions in DTA system. DES should be able to capture and model different types of ATMS which may be deployed within a given scenario, such as Real-Time TRaffic Adaptive Control System (RT-TRACS) technology that is currently being developed by FHWA.

\section{ATIS Modeling}

Information and guidance generation is another major functions of a DTA system. A set of requirements and a framework for guidance generation have been derived and analyzed by (5) and (6). An ATIS service provider may either use the guidance and information generated by a supporting DTA system or may generate guidance and information based on the network states predicted by a DTA system. ATIS information and guidance and can be disseminated to drivers in a number of different ways. The evolution of demand depends on the adopted type of guidance/information dissemination strategy. In order to analyze the DTA system assumptions and modeling features, it is imperative that the DES be able to simulate different types of ATIS deployment differing in services offered, localities covered, and applying different guidance and information generation and dissemination strategies. 


\section{Existing Simulation Models and Their Limitations}

No single traffic simulation system to date meets all of the requirements identified in the previous sections of this paper. This is partly due to the fact that DTA system requirements were only identified in the past few years, see for example (6), and (7). Another reason for the lack of effort in developing a DTA evaluation system can be attributed, in part, to the complexity of the problem, the need for tremendous amounts of computational resources, and difficulty in forming multi-disciplined development teams that incorporate expertise in the areas of traffic engineering, operations research, control theory, software engineering, and high performance computing, to name just a few. However, several simulation models do exist that meet some of the requirements identified in this section. Some of them are discussed briefly in the following paragraphs.

INTEGRATION (8) is a microscopic, assignment based traffic simulation model that supports integrated modeling of freeways and arterials. Individual vehicles are moved on paths (either predetermined or dynamically changed - based on emerging traffic conditions). Pre-timed, actuated, ramp metering, controls are modeled. Re-routing of traffic occurs under incident conditions. The driver/vehicle population is divided into five subgroups based on guidance information source, routing objective and/or routing logic, e.g., vehicle type 1 are those that are assigned a set of externally calculated routes, vehicle type 2 are those that have access to comprehensive instantaneous databases. Major limitations of INTEGRATION are that: it does not model transit; trip generation, mode choice, departure time choice; limited surveillance systems modeling; incidents cannot be stochastically generated; limited ATIS modeling. Another major limitation is that it is a proprietary software.

DYNASMART (9) is a mesoscopic, assignment-based, traffic simulation model that supports integrated modeling of freeways and arterials. Individual vehicles or group of vehicles are moved on paths (chosen based on the driver class) and based on macroscopic flow models. Controls are modeled as capacity restrictions. Traffic gets re-routed under incidents. The model assumes that all the drivers can be classified as either SO, UO, or BR. The major limitations of DYNASMART are that it: does not model trip generation, mode choice, surveillance system, and incidents. The model is currently being reengineered..

MITSIM (10) is a microscopic traffic simulation model, with advanced traffic control, route guidance, and surveillance modeling capability. Individual vehicles are moved using car following, lane changing, and traffic control response logic. Travelers route choice behavior is captured in a probabilistic route choice model. Toll booth operations are modeled. The major limitations of MITSIM are as follows: does not model transit, mode choice, and departure time choice. The status of the development is not clear. Testing and evaluation of the MITSIM is currently underway to establish credibility of the model.

CORSIM (11) is a microscopic traffic simulation model with very advanced traffic control and limited surveillance system modeling capability. Individual vehicles (including carpools, trucks, and buses) are moved based on vehicle characteristics, driver aggressiveness, and car-following, lane-changing, and traffic control response logic. This is a public domain software and the source code is available subject to some restrictions. The major limitations of CORSIM are that (i) vehicles take random walk through the network based on turning percentages at crossing intersections, (ii) it does not model route, mode, departure time choices, (iii) it does not model driver responses to information, guidance, and controls, (iv) it does not model probe vehicle surveillance, (v) it does not model VMS, and (vi) it does not model ATIS deployment. 


\section{MODELING FRAMEWORK}

This section discusses a modeling framework which permits the development of alternate ground-truth simulators which are compatible with the DSL and meet the requirements presented earlier. The ground-truth simulator for DTA evaluation presented in the following section is based on this framework. Figure 3 presents the overall view of the framework.

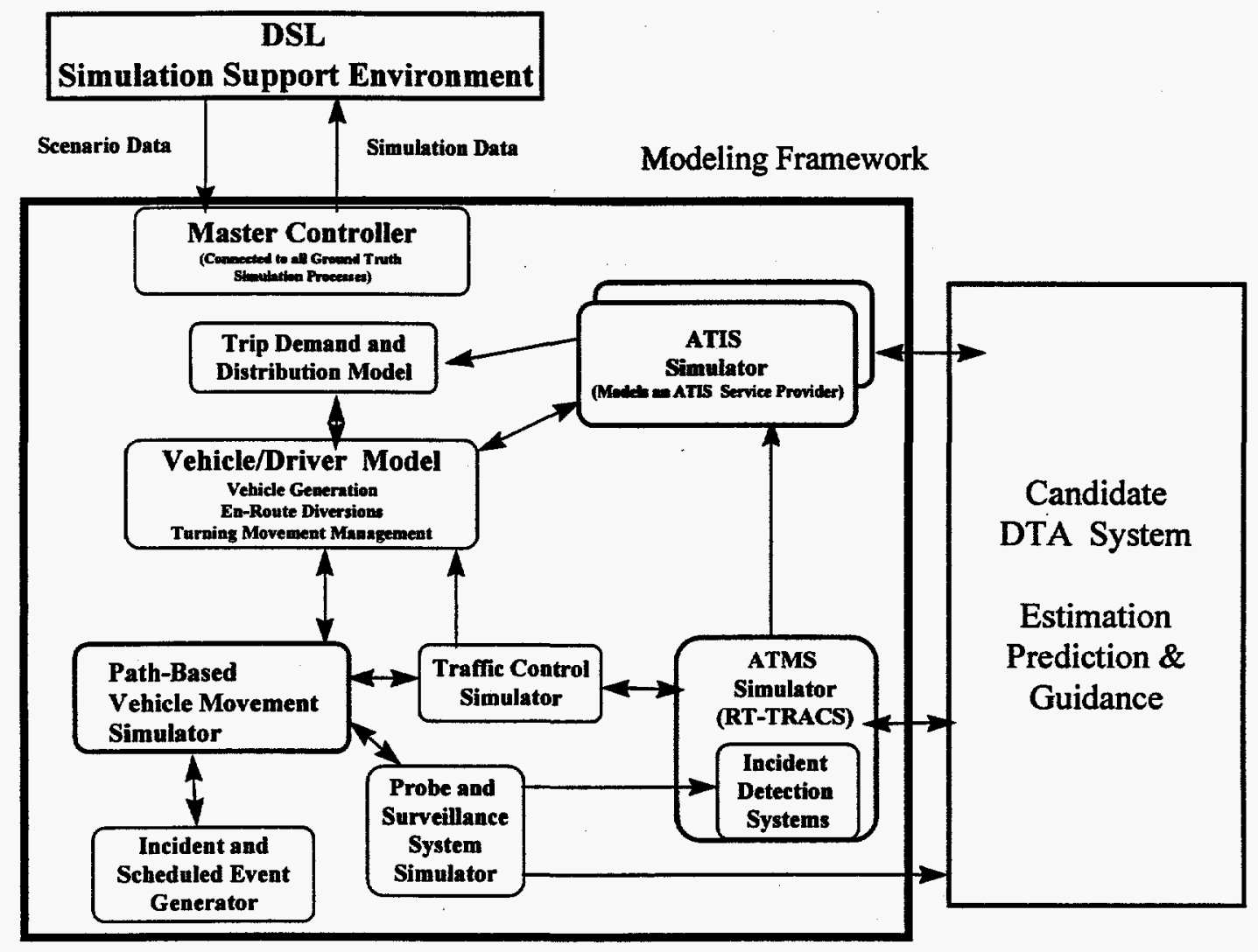

Figure 3: $\quad$ Ground-Truth Simulator Modeling Framework

The large rectangles in Figure 3 represent the modeling framework and its interfaces with the DSL Simulation Support Environment and a candidate DTA system. The rounded boxes represent the various modeling components of the framework.

The requirements discussed earlier can be mapped into each of the framework's components. Requirements regarding ITS system modeling map into the Surveillance System Simulator; Traffic Control Simulator, ATMS Simulator, and the ATIS Simulator. Traveler behavior modeling requirements map into the Trip Demand and Distribution Model and the Vehicle and Driver Manager. Path-based vehicle movement requirements map into the Vehicle Movement Simulator. Incident modeling requirements map into the Incident and Scheduled Event Simulator. Transportation network representation requirements map into all of the components. 
The framework enables ground-truth simulators to be assembled from plug-replaceable components. Any model can be used as a component within this framework provided that it meets the requirements of that component and provides the necessary interfaces. That is, both legacy and independently developed software can be integrated within the framework. Potentially models that can be integrated include pre-trip and en-route choice models, trip demand and distribution models, and alternate vehicle movement simulators.

Currently the framework permits ATMS, ATIS, and surveillance system components. The framework permits later extensions to allow for the modeling of other ITS components and services (e.g., Emergency Management Service, Electronic Toll Collection system (ETC), and Commercial Vehicle Operations (CVO) services).

The framework presented in Figure 3 supports both sequential and distributed implementations. The next section presents a distributed ground-truth simulator designed within this framework.

\section{GROUND-TRUTH SIMULATOR DESIGN}

This section presents the design of the DES ground-truth simulation system. This system is part of ORNL's DSL presented earlier and is supported by DSL simulation support components as shown (see Figure 2). The ground-truth simulator has been designed as a distributed system to address performance requirements.

In our distributed implementation, each modeling component of Figure 3 is implemented as either a single process or a distributed sub-system. Each distributed component presents itself to the system as a collection of simulated objects. If a legacy code is used to implement any of the distributed subsystems, object-oriented "wrappers" can be added to the legacy code so that the legacy system appears as a collection of objects to the distributed simulation system. In this approach, objects of the same kind are generally grouped in the same process. This helps meet performance requirements since frequently invoked operations on objects can be better optimized for performance if all of the objects are in the same process.

The resulting distributed system design provides a natural framework which

- provides opportunities for the optimization of distributed performance

- provides for object-oriented software within each distributed component

- enables the utilization of legacy software systems

The following sub-sections describe each of the components presented in Figure 3 as they would be implemented in a distributed system. They are described in terms of the types of objects that they contain, the roles that they play in the distributed simulation and their interfaces.

\section{Master Controller}

This process contains objects representing the distributed simulation and its components. It is responsible for the management of the different processes that make up the distributed simulation and for establishing external and internal linkages. Its role includes

- ensuring that all the processes required by the DES are running and ready when needed

- coordinating the loading of scenario data required by different DES processes 
- starting, pausing, and stopping the execution of the distributed simulation system

- controlling the generation of simulated output and its collection by the DSL Simulation Support Environment

Because of its managerial role this module has interfaces to all of the other DES processes (not shown in Figure 3), the DTA system being tested, and the DSL Simulation Support Environment.

\section{Trip Demand and Distribution Model}

This sub-system contains objects which represent models of travel demand, mode-choice, trip distribution, and the effects of ATIS information on pre-trip decision making behavior.

The role of this model is to dynamically generate trips and assign origins, destinations, and departure times to those trips. This process will be based on prescriptive and descriptive ATIS information, as well as historical data.

During each time step, the Trip Demand and Distribution model will receive from the ATIS simulators any ATIS pre-trip information that is available to various travelers at that time. It will also receive from the Vehicle Movement Simulator information regarding the size of vehicle queues that exist on network entry links so that it can account for situations in which demand is overloading network entry points.

The model generates individual vehicle departures that will occur during each simulated time step. Each departure will be tagged with vehicle and driver class information and any available pre-trip route selections. These departures are sent to the Vehicle and Driver Manager.

\section{Vehicle and Driver Manager}

This sub-system manages vehicle and driver objects which are also contained within the Vehicle Movement Simulator. The driver objects contained in this sub-system principally represent driver enroute diversion behavior whereas the corresponding driver objects in the Vehicle Movement Simulator contain turning movement lane changing, and other types of behavior. The Vehicle and Driver Manager vehicle objects principally represent the vehicle's ATIS equipment whereas the vehicle objects in the Vehicle Movement Simulator are used to represent the motion of the same vehicle within the transportation network. Vehicle and Driver Manager vehicle objects contain ATIS in-vehicle equipment objects and in-vehicle ATIS display objects. The role of the Vehicle and Driver Manager is twofold (i) it models the en-route diversion behavior of drivers and (ii) it generates and manages vehicle and driver objects.

During each simulated time step, the Vehicle and Driver Manager receives any available prescriptive and descriptive ATIS guidance and information from the ATIS simulator, local traffic conditions from the Vehicle Movement Simulator, and VMS data from the Traffic Control Simulator. Using this information and the characteristics of the driver's it models the driver's cognitive work load, lanechanging aggressiveness, and route diversion choices.

The Vehicle and Driver Manager receives vehicle departure information from the Trip Demand and Distribution Model and generates vehicles and drivers corresponding to these departures. It then injects these vehicles into the Vehicle-Movement Simulator at the origin of their route during the proper simulation time-step. The correspondence between vehicle and driver objects within the simulation is maintained by notifying the Vehicle Movement Simulator of diversions and changes in lane-changing aggressiveness identified within the Vehicle and Driver Manager. 


\section{Vehicle Movement Simulator}

The Vehicle Movement Simulator contains many different types of objects including transportation network objects, traffic signal objects, vehicle objects, and driver objects. The transportation network objects include networks, links, lanes, nodes, path links, and routes

The Vehicle Movement Simulator's role is to model the motion of vehicles through a specified transportation network whose signals are controlled by the Traffic Control Simulator and to generate and collect all of the vehicle data required for the analysis of simulation runs.

Prior to each simulated time step, the Vehicle Movement Simulator receives the following information from the Vehicle and Driver Manager

- the vehicles and drivers that are entering the simulated network at the current simulation time step

- the paths that new vehicles and diverted vehicles are to follow

- any new levels of lane-changing aggressiveness that are indicated by ATIS information and the current route and driver-observed traffic conditions

The Vehicle Movement Simulator then receives from the Traffic Control Simulator VMS messages that can effect vehicle speeds and the signals that are displayed on each signal face contained within the Vehicle Movement Simulation during the next simulated time step. From the Incident and Scheduled event Generator it receives descriptions of any incidents or events which will start during the next simulated time step.

After the Vehicle Movement Simulation has simulated the movement of all vehicles for the current simulation time step, it sends the Vehicle and Driver Manager

- information regarding any vehicles that have completed or not been able to follow their assigned route

- any changes in the traffic conditions observed by drivers

At this point the Vehicle Movement Simulation sends to the Probe and Surveillance System Simulator and the Traffic Control Simulator vehicle position information required by traffic surveillance system objects, vehicle detectors associated with actuated controllers, and probe vehicle data reporting equipment. It also sends traffic condition data to the Incident and Scheduled Event Generator.

\section{Incident and Scheduled Event Generator}

This process contains incident and incident generator objects. Its role is to generate incidents (modeled in the Vehicle Movement Simulator) based on the current state of the traffic on each link, the links geometry, and weather conditions. The incidents and events may be generated either randomly based on distributions which are conditioned upon relevant traffic conditions, or based on incidents and events scheduled in a scenario.

During every simulated time step, the Incident and Scheduled Event Generator receives traffic condition data from the Vehicle Movement Simulator and sends it objects representing incidents that start during the next simulated time step. 


\section{Surveillance System Simulator}

This sub-system contains objects which represent the traffic surveillance system and the equipment which generates data from probe vehicle observations and/or reports. These objects include wide area surveillance equipment, point-to-point surveillance equipment, and all types of vehicle detector capabilities (e.g., vehicle classification systems).

The role of this system is to simulate the generation of the surveillance and probe vehicle data reports that the DTA system would receive in the simulated ITS deployment.

Prior to each simulated time step, this sub-system receives vehicle position reports from the Vehicle Movement Simulator on an on-demand basis (e.g., sensor objects can indicate their field of view and request that the Vehicle Movement Simulator provide information on vehicles that are currently within that field of view).

The Surveillance System Simulator generates the probe vehicle and surveillance system reports that would be transmitted by the system and sends them to the DTA system under test on an on-demand basis. All reports will be tagged with the type of system which generated the information and the simulated time at which the information was made available.

\section{Traffic Control Simulator}

This sub-system contains objects which represent VMS signs, controllers of all types (including pretimed and actuated controllers) and vehicle detectors that are connected to actuated controllers. Its role is to determine what is to be displayed on the faces of the signals connected to each controller and to manage VMS objects and the dissemination of their messages. Each controller object stores and maintains any required timing plans and timing plan libraries.

Prior to each simulated time step the Traffic Control Simulator will receive vehicle position information so that it can determine the detector outputs that are required by the actuated controller objects. It also receives commands from the ATMS Simulator to switch controller timing plans or to receive timing plans down-loaded from the ATMS simulator. The Traffic Control Simulator provides controller status updates to the ATMS Simulator on demand.

The Traffic Control Simulator then determines the signals to be displayed on each signal during the next simulated time step and sends this information to the Vehicle Movement Simulator. It also sends the Vehicle Movement Simulator VMS messages that affect vehicle speed. VMS messages that affect routing decisions are sent to the Vehicle and Driver Manager.

\section{ATMS Simulator}

The ATMS simulator contains objects which represent wide-area traffic controllers, TMCs, and incident detection and management systems. The role of the ATMS simulator is to model the effect of multiple TMCs and systems such as RT-TRACS and its successors. This includes the modeling of incident detection systems and the generation of VMS displays.

During each time step the incident detector objects contained within the ATMS Simulator receive traffic reports from the Probe and Surveillance System Simulator. The TMC and wide-area controller objects receive traffic-controller state updates from the Traffic Control Simulator. The TMC and wide-area controller objects receive estimated and predicted traffic conditions for the entire network from the DTA system under test on an on-demand basis. 
Based on DTA system outputs the TMC and wide-area controller objects determine new strategies and generate new signal timing plans and offsets for the traffic controller objects contained within the Traffic Control Simulator. ATMS simulator sends these strategies and timing plans to the DTA system. The ATMS simulator also determines the VMS messages to be displayed and send them to the Traffic Control Simulator. The ATMS simulator then generates incident detection reports and traffic controller status reports and sends them to the DTA system under test on an on-demand basis.

\section{ATIS Simulator}

The ATIS simulator contains objects which represent ATIS service providers. The ATIS service providers provide to their subscribers all or part of the information provided to them by the DTA system (and possibly other sources). They may provide the information in different formats, via broadcast or on an on demand basis, and the information may be delayed.

The role of the ATIS simulator is to represent the operation of one or more ATIS service providers serving various subscribers which are represented in the Vehicle and Driver Manager and the Trip Demand and Distribution Manager.

During each time step, the ATIS Simulator receives traffic state estimates and predictions, as well as suggested route guidance from the DTA system. Using this information (and possibly other sources of information), the ATIS Simulator generates subscriber messages and sends them to the Vehicle and Driver Manager and in the Trip Demand and Distribution Model.

\section{RESULTS, FUTURE WORK, AND RESEARCH OPPORTUNITIES}

This paper presents the requirements for a ground-truth simulator that is required for comprehensive and cost-effective DTA systems tests and evaluations within the DSL. These requirements are used to develop a modeling framework which in turn is used in the design of a distributed ground-truth simulator called the DES.

The modeling framework presented in this paper maps DTA evaluation requirements into a natural set of plug-replaceable components supporting the re-use and integration of both legacy and independently developed software. The framework is extendible in that it allows for the modeling of additional ITS components and services at a later date. The framework supports both sequential and distributed implementations.

The DES design presented in this paper is a distributed implementation of the modeling framework. It provides opportunities for the optimization of distributed performance; enables the utilization of legacy software systems; and provides for object-oriented software within each distributed component.

ORNL's DES design is currently being implemented by VIGGEN Corporation as an extension to the capabilities of the current Traffic Research Laboratory (TReL).

\section{Research Opportunities}

The modeling framework presented can be used to evaluate future models (e.g. travel behavior models) within the context of an overall ITS simulation and to comparatively evaluate alternate models which perform the same role.

In addition to DTA system evaluations, the DES can also be used to perform laboratory tests and evaluations of transportation system operations under different ITS deployments. 


\section{ACKNOWLEDGMENTS}

This paper is based on the results of a research project sponsored by the Intelligent Systems Division, Office of Safety and Traffic Operations Research and Development, FHWA. The opinions expressed in this paper are, however, solely those of the authors. The first three authors of this paper have jointly and equally contributed to the preparation of this paper. Many of the authors have benefited from the discussion with Al Santiago and Jim Clark of FHWA, the DTA team members of UTX and MIT, and the TReL/CORSIM team members of VIGGEN Corporation and Kaman Sciences Corporation.

\section{REFERENCES}

1. "Conceptual Design for Real-Time Dynamic Traffic Assignment System," Technical Report ST067-85-TASKC, Center for Transportation Research, The University of Texas at Austin, March 1996.

2. “Task C Interim Report: Conceptual Design and Framework," Intelligent Transportation Systems Program, Massachusetts Institute of Technology, April 1996.

3. "Real-Time Dynamic Traffic Assignment Model Research," Draft Technical Report, Intelligent Transportation Systems Group, Center for Transportation Analysis, Oak Ridge National Laboratory, February 1996.

4. "CORSIM Enhancements Required for DTA System Evaluation and Testing," Technical Report, ITS Group Center for Transportation Analysis, Oak Ridge National Laboratory, January 17, 1996

5. Kaysi, I., Ben-Akiva, M., and Koutsopoulos, H., "Integrated Approach to Vehicle Routing and Congestion Prediction for Real-Time Driver Guidance," Transportation Research Board Record, $1408,1993$.

6. Mahmassani, H., T. Hu, S. Peeta, and A. Ziliaskopoulos, "Development and Testing of Dynamic Traffic Assignment and Simulation Procedures for ATIS/ATMS Applications," Technical Report DTFH61-90-C-00074-FG, prepared for USDOT, 1994.

7. Friesz, T. L., et al. "Dynamic Network Traffic Assignment Considered as a Continuous Time Optimal Control Problem." Operations Research 37, pp. 893-901, 1989.

8. Van Aerde, M., "INTEGRATION: A Dynamic Traffic Simulation/Assignment Model," Paper presented at the IVHS Dynamic Traffic Assignment and Simulation Workshop, FHWA, McLean, VA., 1992.

9. Jayakrishnan, R., Mahmassani, H. S., and Hu, T., "An Evaluation Tool for Advanced Traffic Information and Management Systems in Urban Networks," Transportation Research C, Vol. 2, No. 3, PP 129-147, 1994.

10. Yang, Q., and Koutsopoulos, H., “A Microscopic Traffic Simulator for Evaluation of Dynamic Traffic Management Systems,” Working Paper, M.I.T., 1995.

11. “CORSIM User Manual, Version 1.0,", FHWA, Office of Safety and Traffic Operations R\&D, McLean Virginia, 1995. 